\title{
A 30-Year-Old Man with HIV, Fever, and a Rash
}

\author{
Radhika Shah $^{a}$ Saami Khalifian ${ }^{a} \quad$ Lyn Duncan $^{b} \quad$ Daniela Kroshinsky $^{a}$ \\ Bonnie Mackool ${ }^{\mathrm{a}}$ \\ Departments of a Dermatology and ${ }^{b}$ Dermatopathology, Massachusetts General Hospital, \\ Boston, MA, USA
}

Keywords

HIV · Rash · Fever

\begin{abstract}
Patients who present with papular rashes have a wide differential diagnosis particularly in the setting of immune compromise. A 30-year-old male diagnosed with HIV since 2009, never on antiretroviral therapy, with a nadir CD4 count of $333 \mathrm{cells} / \mathrm{mm}^{3}$ and a current viral load of 44,300 copies $/ \mathrm{mL}$, presented with a diffuse monomorphic papular eruption that began on his trunk and extremities and subsequently spread to the penis and scrotum, sparing the distal acral sites. A thorough infectious workup revealed a positive rapid plasma reagin (RPR) and varicella IgM and IgG antibodies. Interestingly, the patient had been diagnosed and treated for syphilis in the past with a recent downtrending RPR drawn prior to hospitalization. Repeat RPR was elevated and a preliminary histopathology report demonstrated folliculocentric inflammation with lymphocytes, plasma cells, and polymorphonuclear leukocyte predominance supported the diagnosis of syphilis. After receiving intramuscular penicillin $\mathrm{G}$ benzathine, he developed intermittent fevers and new papules. Intravenous (IV) acyclovir was initiated for presumed disseminated varicella given his positive varicella-zoster virus IgM and IgG. However, final pathology results revealed a large spirochete burden. The fevers and rash progression were attributed to the development of a Jarisch-Herxheimer reaction. IV acyclovir was discontinued and he completed a course of intramuscular penicillin $\mathrm{G}$ benzathine. He was also given a course of doxycycline for rectal chlamydia which was diagnosed during hospitalization.

(c) 2018 The Author(s)

Published by S. Karger AG, Basel
\end{abstract}




\section{Case Report}

A 30-year-old male diagnosed with HIV since 2009, never on antiretroviral therapy, with a nadir CD 4 count of 333 cells $/ \mathrm{mm}^{3}$ and a current viral load of 44,300 copies $/ \mathrm{mL}$, anal intraepithelial neoplasia 2, and a history of unprotected sexual contact with multiple partners, presented with fever, night sweats, and a diffuse monomorphic papular eruption that started on his trunk and extremities and subsequently spread to his penis and scrotum and spared his distal acral sites. Given the broad differential for papular lesions in an immunocompromised host, a punch biopsy of the left forearm was performed for histology and bacterial/ fungal/acid-fast bacilli culture. The patient was unsure if he ever had a history of primary varicella.

\section{Diagnosis and Clinical Course}

Varicella-zoster virus (VZV) IgM and IgG were both positive, and he was started on intravenous (IV) acyclovir $10 \mathrm{mg} / \mathrm{kg}$ every $8 \mathrm{~h}$ given the concern for possible disseminated VZV. Direct fluorescent antibody exam was not performed due to the absence of vesicular lesions. A swab of his rectum revealed chlamydia, which was treated with doxycycline for 7 days. Initial histopathologic examination revealed mixed folliculocentric inflammation with lymphocytes, plasma cells, and polymorphonuclear leukocytes and a high burden of intraepidermal and intrafollicular spirochetes. Rapid plasma reagin (RPR) titer was reactive at 1:128 dilution, which was significantly higher than his last RPR prior to admission. The history of immunosuppression, clinical findings, and positive RPR titer with interval increase from prior supported the diagnosis of secondary syphilis. The patient was given one dose of intramuscular penicillin benzathine of 2.4 million units. Disseminated VZV was considered unlikely given the lack of vesicular lesions and IV acyclovir was discontinued. Following penicillin therapy, lesions increased in number on the face and scalp with persistent scaling of the arms, trunk, penis, and scrotum. He had no palmar or plantar lesions or lymphadenopathy but lowgrade fevers continued.

After 4 days of acyclovir therapy, final dermatopathology results confirmed a high spirochete burden with an absence of any herpetic infection (herpes simplex virus or VZV) on biopsy specimen upon immunohistochemical staining (Fig. 1). The development of fevers and

new lesions was attributed to a Jarisch-Herxhiemer reaction. The patient was scheduled to receive the remaining two of three weekly doses of intramuscular penicillin benzathine of 2.4 million units as an outpatient. Follow-up examinations and repeat RPR titers at 6, 12, and 24 months following treatment were scheduled.

\section{Discussion}

Syphilis is a venereal infectious disease caused by Treponema pallidum which spreads hematogenously infecting various organs. Diverse clinical presentations of syphilis pose diagnostic challenges, leading to the disease being called "the great imitator." Sir William Osler underscored this challenge by noting that "the physician who knows syphilis knows medicine."

The disease has three different stages, primary, secondary and tertiary syphilis, all of which present with unique clinical manifestations. After initial exposure, the incubation period ranges from 10 to 90 days before a painless papule with regional adenopathy appears, usually in the genital region. These lesions generally self-resolve in 2-6 weeks. Approximately 
Fig. 1. Right arm punch biopsy: numerous intradermal and intrafollicular spirochetes visualized on immunohistochemistry. a Lowpower view. b High-power view.
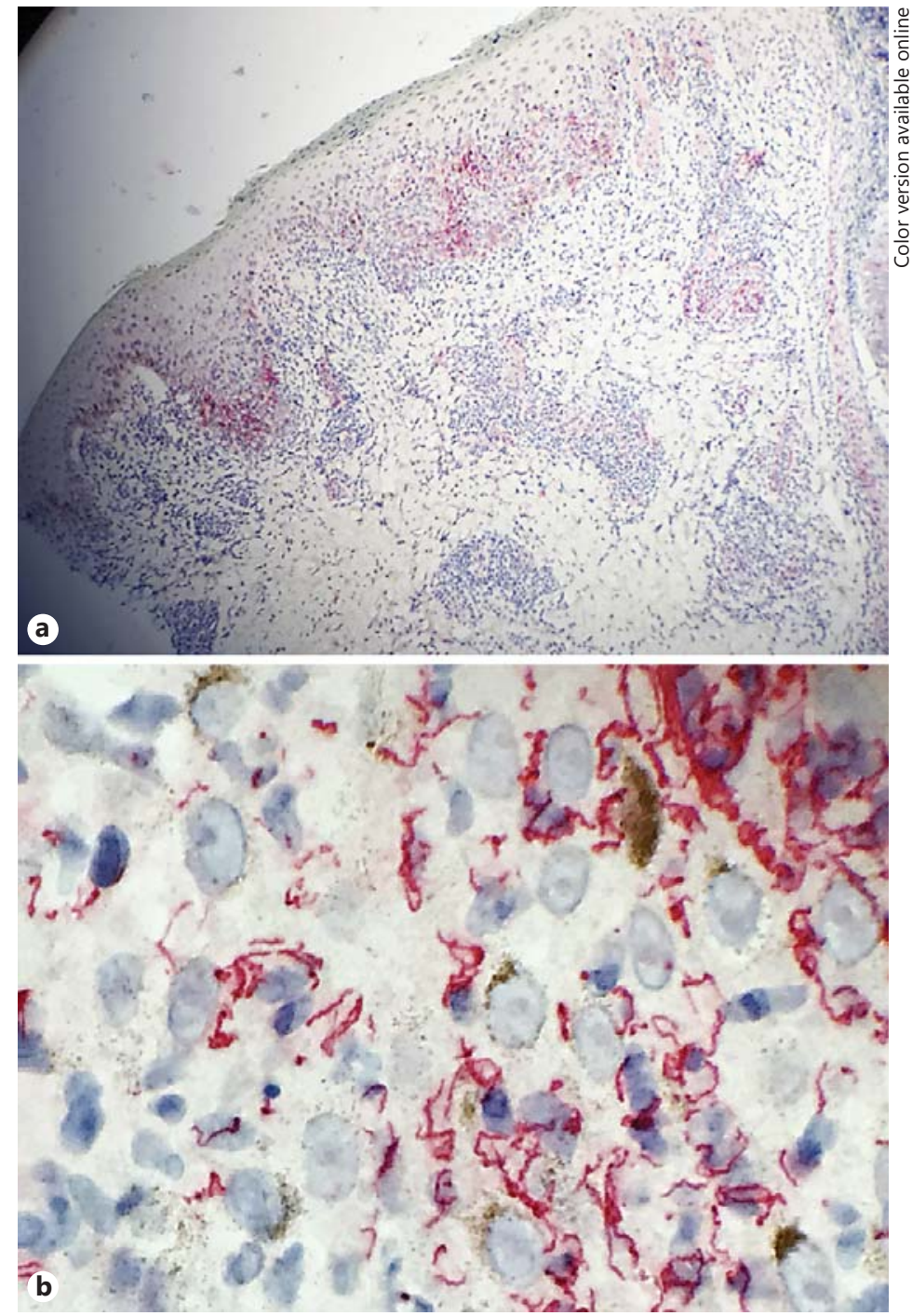

1-2 months after the formation of the initial papule, the patient develops symptoms such as low-grade fever, fatigue, generalized adenopathy, and mucocutaneous rash. This erythematic nonpruritic rash covers the body symmetrically and characteristically involves the hands and the soles of the feet. Skin biopsies of these lesions reveal plasma cells with mononuclear infiltrates. Secondary syphilis can present with an array of symptoms. The development of a diffuse nonpruritic maculopapular rash of the torso, extremities, and specifically palms and soles of the feet is characteristic. These patients may also develop mucosal lesions, alopecia, and generalized lymphadenopathy. Histopathology can reveal either caseating or noncaseasting granulomas with dermal plasma cells and lymphocytes. These cutaneous manifestations appear as a result of hematogenous spread of the spirochetes from the initial chancroid lesions. Patients who have concomitant syphilis with HIV have been reported to develop a wider array of manifestations; these include lesions that resemble eczema, mycosis fungoides, and leprosy. Their morphologies range from papulosquamous, lentricular, pustular to annular [1]. There have been rare reports of secondary syphilis that has presented with vesicular lesions [2]. If secondary syphilis goes untreated, it will progress to tertiary syphilis, which presents with cardiovascular and neurologic symptoms [1]. 
There is a high rate of co-infection of syphilis in patients who are HIV positive. This rate is found to be especially high in patients who are identified as men who have sex with men $[1,3,4]$. Several studies have found that HIV-positive patients with syphilis have a transient increase in HIV viral load and a decrease in CD4 count $[5,6]$.

The diagnosis of secondary syphilis is made through the use of IgM and IgG antibodies, which react with RPR, the Venereal Disease Research Laboratory test, and toluidine red unheated serum test. A biopsy is usually performed to confirm the diagnosis [7]. The treatment of secondary syphilis is a single dose of 2.4 million units of intramuscular penicillin benzathine, although studies have supported the use of three weekly doses of intramuscular penicillin in patients who are co-infected with HIV [3].

A positive RPR of 1:128 dilution in combination with clinical history, in a HIV-positive patient, raised the suspicion of syphilis. His high varicella antibody count led to the consideration of possible herpes folliculitis. Biopsy results confirmed syphilis revealing a high spirochete burden. Development of intermittent fevers with the emergence of new lesions following antibiotic therapy supports the development of a Jarisch-Herxheimer reaction in this patient.

A Jarisch-Herxheimer reaction occurs in more than $50 \%$ of patients who are treated for syphilis [8]. HIV-infected individuals are thought to have a higher incidence than patients who are not infected by HIV [9]. Patients are diagnosed if they develop a fever of $>38.0^{\circ} \mathrm{C}$ and/ or an exacerbation of maculopapular rash within $24 \mathrm{~h}$ of penicillin treatment [9]. Clinical presentation also includes chills, headaches, hemodynamic instability, and myalgias. This reaction is thought to be the result of the destruction of a large number of spirochetes, resulting in a massive inflammatory reaction.

\section{Statement of Ethics}

The manuscript was prepared in compliance with all ethical and confidentiality guidelines and principles.

\section{Disclosure Statement}

The authors have no conflicts of interest to disclose.

\section{References}

1 Goh BT: Syphilis in adults. Sex Transm Infect 2005;81:448-452.

2 Arora S, Dhali TK, Haroon MA: Vesicular syphilid in a seropositive patient. Int J STD AIDS 2013;24:905-907.

-3 Centers for Disease Control and Prevention (CDC): Primary and secondary syphilis - United States, 20032004. MMWR Morb Mortal Wkly Rep 2006;55:269-273.

-4 Burchell AN, Allen VG, Gardner SL, Moravan V, Tan DH, Grewal R, et al: High incidence of diagnosis with syphilis co-infection among men who have sex with men in an HIV cohort in Ontario, Canada. BMC Infect Dis 2015;15:356.

-5 Kofoed K, GerstoftJ, Mathiesen LR, Benfield T: Syphilis and human immunodeficiency virus (HIV)-1 coinfection: influence on CD4 T-cell count, HIV-1 viral load, and treatment response. Sex Transm Dis 2006;33:143-148.

6 Jarzebowski W, Caumes E, Dupin N, Farhi D, Lascaux AS, Piketty C, et al: Effect of early syphilis infection on plasma viral load and CD4 cell count in human immunodeficiency virus-infected men: results from the FHDHANRS CO4 cohort. Arch Intern Med 2012;172:1237-1243.

7 Henaro-Martinez AF, Johnson SC: Diagnostic tests for syphilis. Neurol Clin Pract 2014;4:114-122.

8 Jarisch-Herxheimer reaction. Br Med J 1967;1:384.

-9 Yang C-J, Lee N-Y, Lin Y-H, Lee H-C, Ko W-C, Liao C-H, Wu C-H, Hsieh C-Y, Wu P-Y, Liu W-C, Chang Y-C, Hung C-C: Jarisch-Herxheimer reaction after penicillin therapy among patients with syphilis in the era of the HIV infection epidemic: incidence and risk factors. Clin Infect Dis 2010;51:976-979. 